\title{
LOS BENEFICIOS DEL YOGA Y DE LA MEDITACIÓN PARA EL APRENDIZAJE DE UN INSTRUMENTO MUSICAL
}

\author{
Nerea Olabe ${ }^{1}$ e Cristina Pereira ${ }^{2}$ \\ Escola Superior de Artes Aplicadas do IPCB, Portugal. nerola@gmail.com; \\ ${ }^{2}$ Escola Superior de Educação do IPCB, Portugal. cristina.pereira@ipcb.pt;
}

\begin{abstract}
Resumen. El artículo presenta un trabajo de investigación-acción desarrollado con la clase de violoncello de un conservatorio con la intención de estudiar los beneficios del yoga y la meditación en el aprendizaje de un instrumento y en el manejo de algunos problemas inherentes a los estudiantes de música, como trastornos musculoesqueléticos y ansiedad escénica. Se pretende que los resultados ayuden a mejorar la práctica docente, alejando las enseñanzas musicales del sufrimiento psíquico, emocional y físico que en ocasiones padecen los músicos. Durante el desarrollo de la investigación-acción con los cuatro participantes se recogieron datos de la implicación en el aprendizaje, la ansiedad de los participantes, y se implementó un programa de yoga y meditación. Al finalizar se recogieron nuevamente los datos y se solicitó a los participantes un relato personal sobre su experiencia. El análisis de los datos mostró mejoras significativas en la implicación en el aprendizaje, al mismo tiempo que se redujeron los niveles de ansiedad estado y ansiedad escénica. Se puede concluir que yoga y meditación, como complementos de la formación de los estudiantes de música, pueden ayudar a mejorar su aprendizaje y desarrollar herramientas de afrontamiento para problemas que cohabitan con la profesión.
\end{abstract}

Palabras clave: Meditación Mindfulness; Yoga; Problemas Musculoesqueléticos; Educación Musical; Ansiedad Escénica.

\section{THE BENEFITS OF YOGA AND MEDITATION FOR LEARNING A MUSICAL INSTRUMENT}

\begin{abstract}
The article presents an action research work developed with the cello class of a conservatory with the intention of studying the benefits of yoga and meditation in the learning of an instrument and in the management of some problems inherent to music students, such as musculoskeletal disorders and stage anxiety. The results are intended to help improve teaching practice, distancing musical teachings from the psychological, emotional and physical suffering that musicians sometimes suffer. During the development of the action research with the four participants, data were collected on the involvement in learning, the anxiety of the participants, and a yoga and meditation program was implemented. At the end, the data was collected, again, and the participants were asked for a personal account of their experience. Data analysis showed significant improvements in learning engagement, while state anxiety and stage anxiety levels were reduced. It can be concluded that yoga and meditation, as complements to the training of music students, can help improve their learning and develop coping tools for problems that coexist with the profession.
\end{abstract}

Keywords: Mindfulness Meditation; Yoga; Playing-Related Musculoskeletal Disorder; Music Education; Performance Music Anxiety.

\section{INTRODUCCIÓN}

La educación de hoy demanda algo más que el mero aprendizaje de los contenidos curriculares. Se ha vuelto indispensable cuestionar seriamente la manera de educar y enseñar a nuestro alumnado. Es el momento de asumir responsabilidades y de cultivar otras habilidades como el autoconocimiento, la regulación emocional, habilidades interpersonales, la tolerancia, el respeto y el amor hacia uno mismo y hacia los demás, e incluso, ciertas 
habilidades cognitivas, entre las que destaca la atención, que se está degradando desde la omnipresencia de las nuevas tecnologías.

Ser músico profesional puede transformarse en una carrera llena de estrés, con ansiedad escénica; inestabilidad por causa de la imprevisión e irregularidad de horarios de ensayos, conciertos o viajes; además de diferentes trastornos musculoesqueléticos relacionados con la práctica producto de los movimientos repetitivos realizados durante horas (Brandfonbrener \& Kjelland, 2002).

Tal vez, cuando el público de los conciertos ve a un músico actuar se lo imagina como una experiencia maravillosa, sin embargo, aunque pueden ser conscientes de la enorme cantidad de trabajo, motivación y dedicación que se requiere para convertirse en un músico profesional, pocos se darán cuenta de las dificultades y angustia que puede estar asociada con el aspirante a músico (Stoeber \& Eismann, 2007).

Existen estudios que estiman que alrededor del $69 \%$ de los músicos siente ansiedad escénica musical $^{1}$ y que más del $87 \%$ sufre algún problema musculoesquelético (Khalsa, Butzer, Shorter, Reinhardt, \& Cope, 2013). Al mismo tiempo, no son solo los músicos profesionales los que padecen estos trastornos, sino que la propia presión de las clases regulares, la intensa práctica individual, los recitales, concursos, etc., también pueden provocar problemas musculoesqueléticos y fatiga emocional en un gran número de estudiantes de música (Khalsa, Shorter, Cope, Wyshak, \& Sklar, 2009), además de desarrollar ansiedad escénica (Cox \& Kenardy, 1993).

Es esencial, como formadores, dejar de considerar estos problemas del mundo de la música como algo inherente a la profesión para plantearse cómo conseguir desarrollar un aprendizaje más saludable a largo plazo, tanto físico como mental y emocionalmente para los futuros profesionales.

Leer sobre la experiencia del excepcional violinista Yehudi Menuhin con el yoga es muy alentador al poder comprobar cómo éste supuso un gran cambio en su vida como músico. Tanto es así que denominaba al que fue su maestro de yoga, lyengar (2017), como "su mejor

\footnotetext{
1 Término usado por Dianna Kenny en sus artículos y su libro The psychology of music performance anxiety (2011). La traducción del término a castellano aparece en el artículo de Zarza-Alzugaray, Casanova-López y RoblesRubio, Relación entre la ansiedad escénica, perfeccionismo y calificaciones en estudiantes del Título Superior de Música (2016).
} 
profesor de violín" (lyengar, 2017, p. 11). Por lo que, si a alguien de su nivel y ya adulto, le aportó una experiencia de crecimiento, tanto a nivel postural como interpretativo y musical, qué no será posible si esta práctica es introducida desde la iniciación instrumental.

Por otro lado, diversas investigaciones han estudiado los efectos positivos de la práctica regular de la meditación en la concentración (Brown \& Ryan, 2003) y la reducción de la ansiedad escénica en músicos (Chang, Midlarsky, \& Lin, 2003). Si para tener un buen estado físico las personas hacen diferentes tipos de ejercicio, para tener un estado mental saludable, estable y fuerte se entiende que también se debe practicar ejercicio, y este es el que se realiza con la meditación. Se podría decir, por tanto, que "la meditación es el adiestramiento de la mente" (Carrasco, 2016, p. 12).

Como docente se debe confiar en la importancia de estas disciplinas sobre las que existen estudios sobre su relevancia para la formación de un músico (Xu, 2010; Adams, 2012; Khalsa, Butzer, Shorter, Reinhardt, \& Cope, 2013; Zelazo \& Lyons, 2012). Se espera que este estudio sea un incentivo para incluir estas prácticas en las enseñanzas musicales, para con ello mejorar el aprendizaje del alumnado y, al mismo tiempo, la práctica docente. Se ha comprobado que los programas psicoeducativos proporcionados al principio de la carrera musical pueden desarrollar la capacidad de bienestar psicológico y la resiliencia en las dimensiones positivas del bienestar (Steyn, Steyn, Maree, \& Panebianco-Warrens, 2016).

El yoga, junto con el mindfulness, la psicología positiva, los programas de educación emocional o las técnicas de relajación son herramientas que pueden ayudar a transformar vidas o, cuanto menos, la manera de vivirla. Las artes, y en concreto la música, ofrecen también oportunidades en este sentido; son experiencias que de repetirlas pueden resultar habituales, obvias o triviales, pero que se basan en una forma especial y diferente de percibir y entender el mundo. La música compromete a la totalidad de la persona. Joaquín Solé (Orozco \& Solé, 2000, p. 83) afirma en su libro que "el verdadero instrumento no es el violín, la batería o la guitarra, sino nuestro cuerpo, que debemos controlar y preparar para una tarea que exige muchas horas de trabajo y dedicación". Por lo que la relación entre las diferentes áreas se crea de una manera natural.

En el presente, estas disciplinas, junto con la técnica Alexander y el método Feldenkrais, son parte esencial de la formación de cualquier músico en un gran número de países (Olson, 2009), sin embargo, en Portugal prácticamente no se ha realizado ninguna experiencia al 
respecto, se considera, por tanto, que puede ser un punto de partida para futuras investigaciones en esta área.

\section{PROBLEMÁTICA Y OBJETIVOS DEL ESTUDIO}

Uno de los problemas con los que nos enfrentamos regularmente en las clases instrumentales con el alumnado es su falta de concentración y atención a los detalles, a veces incluso en cuestiones menos sutiles. Por otro lado, se ha podido comprobar que con los años acaban por desarrollar, de una u otra manera, lo que se denomina ansiedad escénica, que en muchos casos lleva a evitar los momentos de estrés e incluso a desistir de seguir una carrera musical.

Al mismo tiempo, a pesar de que la consciencia corporal y la correcta alineación del cuerpo son uno de los pilares más importante de la práctica docente, se ha podido comprobar cómo algunos alumnos acaban desarrollando lesiones. Lo que lleva a plantearse si la consciencia corporal, que es esencial para una buena interpretación instrumental, puede ser desarrollada en paralelo para poder mejorar con ello la salud física del alumnado.

Para la investigación en cualquier área hay muchas preguntas que se plantean ser respondidas, aunque a menudo éstas se pueden resumir en unos pocos temas fundamentales, y extremamente importantes al mismo tiempo. Para quienes se interesan por las etapas iniciales del aprendizaje de un instrumento musical, una de las preguntas más básicas es la que se refiere al modo en que el progreso musical está ordenado y en secuencia, y al hecho de que algunos niños parezcan no sentir esfuerzo por aprender en contraste con aquellos que viven una constante lucha, y al mismo tiempo, observar cómo se desarrolla la relación con el escenario, lugar de trabajo esencial para un futuro músico.

Se cree que el desarrollo de la atención plena, a través de la práctica del yoga y la meditación, en paralelo con el aprendizaje musical puede ayudar a una mejor relación de los músicos con el escenario, la práctica instrumental y mejorar su salud psicológica, física y emocional.

Durante las últimas décadas se han realizado innúmeros estudios (Cox \& Kenardy, 1993; Kenny, Davis, \& Oates, 2004; Zarza-Alzugaray, Casanova-López, \& Orejudo-Hernández, 2016b) sobre la ansiedad escénica y los problemas musculoesqueléticos en la música, pero se han centrado en niveles preprofesionales - estudiantes de enseñanzas superiores - y profesionales - músicos de orquesta, cantantes y solistas. Por otro lado, el análisis de estos problemas desde la perspectiva del niño no ha sido hasta hace relativamente poco una 
prioridad para la psicología. Dado que el aprendizaje instrumental comienza a una edad temprana, es muy probable que estos problemas comiencen su desarrollo también prematuramente.

Tras años de experiencia docente en diferentes centros de enseñanzas musicales de Portugal, con diversos proyectos educativos, se ha podido comprobar cómo la formación reglada está bastante anclada en conceptos de enseñanza obsoletos en muchos casos.

Basándose en los supuestos anteriormente expuestos, este estudio pretende responder a cuestiones relacionadas con el proceso de enseñanza-aprendizaje de un instrumento musical como el violoncello y de la música en general. Los objetivos que dirigen la investigación son los siguientes:

- Mejorar la capacidad atencional del alumnado y su implicación en las actividades de clase individual y colectiva.

- Mejorar la consciencia corporal y, consecuentemente, perfeccionar sus competencias instrumentales y la autoeficacia en el estudio autónomo.

- Reducir la incidencia de problemas musculoesqueléticos.

- Reducir sus niveles de ansiedad escénica y de ansiedad estado.

- Proporcionar herramientas para mejorar el autoconocimiento, la regulación emocional y el bienestar personal y social del alumnado.

- Mejorar el clima del aula y las relaciones alumnado/profesora.

\section{EL ESTUDIO Y LOS SUJETOS ANALIZADOS}

\subsection{Metodología de investigación}

La metodología usada para la investigación es la investigación-acción.

La investigación-acción (IA) es un método de investigación cualitativa basado en la sucesión de planificación, acción observación y reflexión en una realidad concreta, convirtiendo a los protagonistas en investigadores. Tuvo su origen en los Estados Unidos, donde fue concebida y aplicada en un principio por la contribución de varios pensadores, que pertenecían al área de las ciencias sociales y no solo al campo de la educación (Máximo-Esteves, 2008).

Para realizar un proyecto de IA es necesario efectuar un conjunto de procedimientos, de acuerdo con los objetivos de éste: encontrar el punto de partida, reunir la información de 
acuerdo con los patrones éticos, interpretar los datos y validar el proceso de investigación (Máximo-Esteves, 2008).

Por otro lado, existen intentos de relacionar la base contemplativa del budismo, entre ellas el desarrollo de la atención plena, con la IA (Winter, 2003), entendiendo este proceso como una base de observación de la realidad tal cual es, que es el origen de toda IA. Pero apenas hay estudios de mindfulness e IA, pues la mayoría son diseños cuasi experimentales y dejan de lado, aspectos esenciales: colaboración, compromiso, creatividad, curiosidad, reflexión y amabilidad (Winter, 2003).

Eliot (1990) explica que Lewin, uno de los precursores más significativos de esta metodología de investigación, bosqueja un proceso disciplinado de IA que se sitúa en paralelo con la aplicación del método científico en otras disciplinas. Su modelo especifica una espiral de actividades en esta secuencia:

1. Aclaración y diagnóstico de una situación problemática en la práctica.

2. Formulación de estrategias de acción para resolver el problema.

3. Implantación y evaluación de las estrategias de acción.

4. Aclaración y diagnóstico posteriores de la situación problemática (y así sucesivamente en la siguiente espiral de reflexión).

Para concretar el tipo de IA, se parte de los modelos clásicos (Goyette \& Léssard-Hebert, 1988; Elliot, 1990) y de elementos de IA relacionadas con el mindfulness (López-González, 2010). El resultado es:

- Observación de la realidad.

- Idea general.

- Planificación del programa.

- Realización del programa (formación y realización de las actividades experimentales).

- Análisis de los resultados.

- Seguimiento y conclusiones.

\subsection{Técnica de recogida de datos}

\subsubsection{Observación directa en clase}

Se realiza el seguimiento en las clases instrumentales con notas de campo a lo largo del proceso de estudio, poniendo especial atención al comienzo y al final de la aplicación del 
programa. El objetivo de esta observación es evaluar las tensiones o problemas físicos asociados con la práctica instrumental de cada participante, dado que ninguno muestra ninguna patología. Al mismo tiempo, valorar la consciencia corporal adquirida, atendiendo a su capacidad de respuesta y creatividad antes los desafíos físicos y musicales del instrumento.

\subsubsection{Implicación del niño. LIS-YC}

Para evaluar la implicación de los participantes durante las clases de instrumento, sin interferir en el normal funcionamiento de la clase, se opta por el instrumento Leuven Involvement Scale for Young Children (LIS-YC), concebido por el profesor Ferre Laevers para el Proyecto EXE en Bélgica (Laevers, 1994, citado en Bertram \& Pascal, 2009). Esta herramienta mide el nivel de implicación que el niño muestra en las actividades que está realizando (Bertram \& Pascal, 2009), siendo un 1 la puntuación más baja y un 5 la puntuación más elevada. Será medido antes del comienzo del estudio, a la mitad y al final.

\subsubsection{Escala de Ansiedad de Zung (ZAS)}

Dado que en el momento del estudio no han sido traducidas al portugués y validadas en Portugal, ni en población adulta ni en niños o jóvenes, las escalas más reconocidas a nivel internacional para medir la ansiedad escénica: Performance Anxiety Questionarie (PAQ) desarrollado por Cox y Kenardy (1993), Kenny Music Performance Anxiety Inventory (K-MPAI) desarrollado por Kenny et al. (2004), se decide recurrir a otro instrumento, aunque menos específico, si igual de válido para medir la ansiedad estado en el momento considerado de estrés. Se elige la Escala de Ansiedad de Zung (1971), validada ampliamente con la población portuguesa (Rodrigues, 2014). Esta escala evalúa la ansiedad estado, no la ansiedad rasgo, por tanto, permite evaluar la reacción situacional de ansiedad y no la ansiedad como un rasgo de personalidad. El cuestionario recoge 20 ítems que son valorados de 1 a 4 , ofreciendo un resultado que puede oscilar entre 20 , nada de ansiedad, hasta 80 , un nivel altísimo de ansiedad. Se considera que un valor igual o superior a 40 representa un indicador de patología asociada a la ansiedad. Este instrumento será usado para medir el nivel de ansiedad antes de momentos de estrés como conciertos o pruebas. Se recogerán datos antes, a la mitad y tras la aplicación del programa de yoga y meditación. 


\subsubsection{Relato sobre la experiencia personal}

Para evaluar las sensaciones de cada uno de los participantes se solicita, tras finalizar la aplicación del programa, que realicen un relato sobre la experiencia que la práctica de yoga y meditación les ha proporcionado. No se da ningún guión específico ni se piden ítems que nombrar, simplemente se solicita que relaten lo que han experimentado durante las clases y cómo ha influido esto en su día a día. Lo redactan y entregan en el mismo momento.

\subsection{Participantes}

La muestra está formada por un total de 4 alumnas/os (1 niño y 3 niñas) de violoncello de entre 10 y 12 años, que asisten a clases de música en el Conservatorio Regional de Castelo Branco. Procedentes mayoritariamente de familias de nivel socioeconómico y cultural medioalto. Todos están aprendiendo violoncello como instrumento principal. Llevan entre 1 y 3 años de aprendizaje instrumental.

\subsection{Procedimiento}

Fase I. Observación de la problemática, ideas generales y planificación del programa de IA.

Fase II. Se procede a la selección del alumnado que participará en el estudio. Previamente a su participación se solicita el consentimiento por parte de padres, madres o tutores, al cual todos acceden. Los estudiantes no recibieron ningún tipo de recompensa por su participación.

Fase II. Una vez elegido el grupo de alumnos se procede a la evaluación pretest de los niveles de ansiedad y de implicación en el aprendizaje mediante la aplicación de los instrumentos específicos. Una vez obtenida dicha medición inicial, se comienza con la aplicación del programa de entrenamiento en meditación y yoga en las instalaciones del Conservatorio.

Fase IV. El programa de yoga y meditación se lleva a cabo a lo largo de los meses de noviembre de 2017 hasta junio de 2018, con una frecuencia de una sesión semanal grupal de cuarenta y cinco minutos de duración cada una. La actividad se efectúa antes de la clase instrumental del alumnado. Durante la aplicación del programa se procede a la recogida de datos con las diversas herramientas en el mes de marzo de 2018.

Fase V. Al finalizar el programa se procede a evaluar nuevamente los niveles de ansiedad e implicación durante el aprendizaje, con los mismos instrumentos empleados antes y durante. Se solicita también un relato personal a los participantes sobre su experiencia en las sesiones. 
Fase VI. Análisis de resultados.

Fase VII. Seguimiento y conclusiones.

\section{DE LOS DATOS AL ANÁLISIS DE LOS RESULTADOS}

El análisis de los diferentes instrumentos de recogida de datos se ha elaborado de manera individualizada, teniendo en cuenta el grupo tan reducido de participantes, para poder determinar las variaciones en cada uno de los casos.

\subsection{Observación directa en clase}

Las notas de campo recogidas durante la clase instrumental semanal a lo largo del estudio para su análisis posterior han arrojado pocos datos significativos. Todos los individuos implicados carecían de trastornos musculoesquelético al principio o al final, por lo que no ha podido evaluarse si existía algún efecto positivo al respecto. Sí ha podido observarse una mayor seguridad a la hora de saber qué movimientos eran necesarios para realizar algunas acciones en el instrumento, aunque esta mejoría puede estar influida por el aprendizaje natural del instrumento, por tanto, no es un dato concluyente.

En general se puede observar que los pequeños problemas de postura corporal, como por ejemplo tensiones en los hombros o cuello, han mejorado a lo largo del año, y los individuos han aprendido a autocorregirse. Mejorando con ello la consciencia corporal para el aprendizaje de un instrumento tras una intervención basada en el yoga y/o la meditación, como han estudiado diferentes investigaciones (Brandfonbrener \& Kjelland, 2002; Khalsa \& Cope, 2006; Adams, 2012). Por otro lado, se observa que la motricidad fina, esencial para la interpretación de un instrumento musical, no ha sufrido ninguna variación.

A pesar de estas observaciones, no es posible concluir que las mejorías apreciadas en la consciencia corporal sen fruto de la aplicación del programa de yoga y meditación, y no del aprendizaje instrumental desarrollado durante las clases de violoncello y el estudio autónomo.

\subsection{Implicación del alumnado. LIS-YC}

Según los datos (Tabla 1) de la evolución de la implicación en las actividades, aquellos alumnos que estaban en un nivel inferior han aumentado tras el programa de yoga y meditación. 
Al mismo tiempo, se puede observar cómo ha evolucionado mínimamente en aquellos participantes que presentaban un nivel bastante elevado. A pesar de todos presentar una ligera mejoría, no es suficientemente relevante para ser tenida en cuenta en el estudio.

Tabla 1. Implicación en la actividad del niño (1-5)

\begin{tabular}{cccc}
\hline & $\begin{array}{c}\text { Nivel anterior a la aplicación } \\
\text { del programa }\end{array}$ & $\begin{array}{c}\text { Nivel durante la } \\
\text { aplicación del } \\
\text { programa }\end{array}$ & $\begin{array}{c}\text { Nivel posterior a la } \\
\text { aplicación del } \\
\text { programa }\end{array}$ \\
\hline Alumna 1 & 4,17 & 3,67 & 4,33 \\
\hline Alumna 2 & 2,33 & 3 & 3,33 \\
\hline Alumna 3 & 4,17 & 4,33 & 4,33 \\
\hline Alumno 4 & 4,17 & 3,83 & 4,33 \\
\hline Media grupo & 3,71 & 3,71 & 4.08 \\
\hline
\end{tabular}

La poca evolución puede ser debida a que, como ha podido ser constatado según la literatura estudiada, existen evidencias de que la población infantil no clínica presenta una evolución menor con los programas de atención plena, en relación con la población clínica (Zoogman, Goldberg, Hoyt, \& Miller, 2015), consecuencia clara del hecho de la población clínica tener un mayor margen de mejoría al padecer en origen una patología. Por otro lado, la única participante (Alumna 2) que sí presentaba niveles insuficientes durante la actividad al inicio del estudio ha desarrollado una mejoría evidente, un nivel completo según la escala LIS-YC, al finalizar el programa de yoga y meditación.

Al observar la media del grupo de la escala LIS-YC se puede concluir que ha mejorado ligeramente, encontrándose el grupo claramente en el nivel 4 al finalizar el programa, cuando al principio se situaba ligeramente por debajo.

Estos resultados apoyan la hipótesis de que un programa de yoga y meditación basado en la atención plena puede mejorar el proceso atencional de los estudiantes (Alajmo, Jiménez, \& Gantiva, 2013; Cavanagh, et al., 2013; Harne, 2017) mejorando con ello su implicación en la actividad durante el aprendizaje.

\subsection{Escala de Ansiedad de Zung (ZAS)}

Al observar los datos (Tabla 2) de los resultados obtenidos por los sujetos (Alumna 2 y 3 ) que mostraban altos niveles de ansiedad al inicio del estudio, se puede concluir que existe una clara reducción de los niveles de ansiedad escénica, saliendo incluso de los niveles considerados patológicos. 
Este resultado apoya la hipótesis planteada al principio de la investigación de que un programa de yoga y meditación podría ayudar a reducir los niveles de ansiedad escénica, y al mismo tiempo corrobora los resultados que han obtenido otros estudios en el área (Lin, Chang, Zemon, \& Midlarsky, 2008; Farnsworth-Grodd, 2012; Adams, 2012; Khalsa, Butzer, Shorter, Reinhardt, \& Cope, 2013; Collyer, 2018). Stern, Khalsa y Hofmann (2012) sugieren que el yoga puede suponer una prometedora ayuda para los estudiantes de música que padecen ansiedad escénica, y al mismo tiempo Farnsworth-Grodd (2012) indica que la atención plena y la autorregulación, desarrolladas por la meditación, pueden ayudar a reducir los niveles de ansiedad escénica, afirmaciones que son confirmadas por los resultados del presente estudio.

Tabla 2. Resultado del ZAS para la ansiedad escénica

\begin{tabular}{cccc}
\hline & $\begin{array}{c}\text { Ansiedad escénica } \\
\text { anterior a la aplicación } \\
\text { del programa }\end{array}$ & $\begin{array}{c}\text { Ansiedad escénica } \\
\text { durante la aplicación } \\
\text { del programa }\end{array}$ & $\begin{array}{c}\text { Ansiedad escénica } \\
\text { posterior a la aplicación } \\
\text { del programa }\end{array}$ \\
\hline Alumna 1 & 24 & 27 & 35 \\
\hline Alumna 2 & 49 & 45 & 31 \\
\hline Alumna 3 & 45 & 39 & 38 \\
\hline Alumno 4 & 26 & 30 & 32 \\
\hline Media grupo & 36 & 35,25 & 34 \\
\hline
\end{tabular}

Por otro lado, el resultado de evolución de aquellos (Alumna 1 y Alumno 4) individuos que mostraban niveles reducidos de ansiedad al inicio del estudio ha sido el contrario al de aquellos individuos que presentaban una clínica evidente, aumentando en este caso en menor o mayor medida sus niveles de ansiedad al finalizar el estudio.

Este resultado puede deberse a que, como explican Zoogman et al. (2015) en su estudio, la población pediátrica que no padece una patología puede no evidenciar mejorías por la implementación de un programa basado en la atención plena, ya que en la población infantil y adolescente se ha comprobado que ayuda con la reducción de patologías, pero no a establecer hábitos saludables en aquellos pacientes no clínicos. Inclusive, como nos indica White (2012), puede aumentar sus niveles de ansiedad al ser confrontados con pensamientos y emociones de los que aún no tenían consciencia y para los que no poseen las herramientas suficientes de gestión emocional.

Este resultado puede suponer un fallo del programa de yoga y meditación al ser aplicado en población no clínica, generando con ello un problema donde no existía. Puede, al mismo tiempo, ser debido al resultado habitual de la presión de un curso lectivo, durante el cual los alumnos son confrontados con mayor presión conforme avanza el año, traduciéndose en una 
mayor ansiedad ante situaciones estresantes, debido a la acumulación vital. Khalsa y Cope (2006) encontraron que los estudiantes del grupo de intervención de yoga, a pesar de reducir la ansiedad escénica durante y después del programa, mostraron al mismo tiempo un aumento de la ira y de la fatiga, y un descenso del vigor. Concluyeron que estos cambios en el estado de ánimo podían estar generados por el intensivo programa de estudios musicales en el que los individuos se encontraban inmerso durante la investigación, lo que determina que una intervención basada en el yoga y la meditación ayuda a mitigar los factores asociados a los duros planes de estudios, pero no consigue hacer desaparecer todos los síntomas asociados a la presión académica.

\subsection{Relato sobre la experiencia personal}

El análisis de los datos se ha basado en un análisis de contenidos (Bardin, 2008). En el contexto del presente artículo se transcriben apenas algunas ideas centrales destacadas por el alumnado: "usé ejercicios aprendidos durante las clases en otros momentos de estrés, y aunque me ayudó a calmarme, los nervios en realidad no pasaron" (Alumna 1), "las clases me han ayudado a relajar bastante en algunas situaciones de estrés como antes de las pruebas de instrumento, (...) también lo he usado en otras situaciones en las que ha ayudado" (Alumna 3), "si no fuese por las clases me habría encontrado más nerviosa en algunos momentos" (Alumna 2), "he estado más relajada los lunes después de las clases, mientras que los demás días no me sentía así" (Alumna 3).

Destaca que han sentido lo aprendido en las clases de yoga y meditación como una herramienta para ayudar en los momentos de mayor tensión emocional y estrés.

\section{CONCLUSIONES}

El objetivo de este estudio era entender cómo influye un programa de yoga y meditación en el aprendizaje de un instrumento musical, concretamente el violoncello, y poder con ello mejorar la práctica docente. Estas disciplinas han adquirido gran importancia en los últimos años, debido tal vez al ritmo frenético de las sociedades globalizadas, como nos indican Simón (2011) y Thich Nhat Hanh (Hanh \& Plum Village, 2015).

La elección de la metodología para la realización del estudio ha revelado ser adecuada y pertinente dado que ha permitido la operacionalización de una intervención pedagógica que consideramos innovadora, al mismo tiempo que la monitorización de los datos recogidos. Los 
procesos cíclicos de planificación, observación y evaluación demostraron ser fundamentales para el análisis realizado. Realzar además el hecho de la triangulación y secuenciación en la utilización de las diversas técnicas de recogida de datos nos ha permitido la comparación de los resultados y, consecuentemente, una validación cualitativa en el análisis final.

Con relación a la atención e implicación en las actividades de aprendizaje se puede afirmar que aquellos individuos que presentaban una dificultad mayor han mejorado significativamente tras el programa de yoga y meditación. Por otro lado, aquellos individuos que no presentaban problemas relevantes y mostraban una atención e implicación alta, han mejorado solo levemente, no considerándolo suficientemente significativo a nivel individual.

En cuanto a la consciencia corporal, no se puede concluir si las mejoras observadas durante el curso se han debido al normal aprendizaje instrumental, o han sido influidas por el programa de yoga y meditación. Al mismo tiempo, no se ha podido valorar la influencia del yoga y la meditación en los problemas musculoesqueléticos, dado que ninguno de los participantes mostraba patologías al inicio del programa. Si se puede destacar que no ha existido ninguna incidencia a lo largo del estudio de sintomatología relacionada con problemas musculoesqueléticos derivados de la práctica, lo que puede ser consecuencia del programa de yoga y meditación, aunque no son resultados concluyentes.

Al mismo tiempo, si se han hallado resultados concluyentes en relación con la ansiedad escénica de los participantes. Se puede afirmar en primer lugar, que aquellas alumnas que mostraban unos niveles altos de ansiedad escénica han reducido muy significativamente sus niveles hasta niveles inferiores a lo considerado patológico. Este resultado apoya lo observado para el caso de la ansiedad en general por las investigaciones de Epply, Abraham y Shear (1989), Kabat-Zinn, et al. (1992), Miller, Fletcher y Kabat-Zinn (1995), Galantino, Galbavy y Quinn (2008), Beaucheim, Hutchins y Patterson (2008), Alajmo, Jiménez y Gantiva (2013), Gallego, Aguilar-Parra, Cangas, Langer y Mañas (2017), Hoge, et al. (2015), Mañas, Franco, Cangas y Gallego (2011), entre otros, y para el caso de la ansiedad escénica en particular por los estudios de Chang, Midlarsky y Lin (2003), Lin, Chang, Zemon y Midlarsky (2008), Khalsa, Shorter, Cope, Wyshak y Sklar (2009), Khalsa, Butzer, Shorter, Reinhardt y Cope (2013), Xu (2010); Stern, Khalsa y Hofmann (2012), Farnsworth-Grodd (2012), Adams (2012) y Collyer (2018). Aunque es posible que los progresos se hayan visto influidas por la motivación de participar en las actividades de yoga y meditación para poder mejorar sus estados habituales de ansiedad. 
Por otro lado, los individuos que comenzaron con bajos niveles de ansiedad escénica han visto aumenta la misma al final del estudio, a pesar de continuar dentro de los niveles considerados de normalidad. Este dato concuerda con los hallazgos de Zoogman et al. (2015) tras el metaanálisis de los estudios que analizan el efecto de la atención plena en la reducción de la ansiedad y que sugiere que en la población joven un programa basado en el mindfulness muestra mayor efecto de la reducción de la sintomatología negativa que el aumento del funcionamiento positivo. Debido, tal vez, a que las muestras clínicas tienen un mayor margen de mejora segnificatiba que las muestras no clínicas, dada la sintomatología basal más severa. Otras terapias a corto plazo, como la terapia cognitivo-conductual o la psicoterapia interpersonal, también han mostrado mejores resultados positivos para los sujetos con sintomatología basal más severa (Zoogman, Goldberg, Hoyt, \& Miller, 2015). Estos resultados negativos pueden deberse a diversos factores: la influencia de la alta ansiedad percibida en el entorno y/o experiencias de conciertos específicos con resultados negativos (Kenny \& Osborne, 2006), la exarcerbación del perfeccionismo y la ansiedad que genera (Stoeber \& Eismann, 2007) o la mayor conciencia adquirida de la ansiedad y los sentimientos asociados (White, 2012). Al no existir un grupo de control para contrastar los resultados, resulta imposible conocer si el programa del estudio ha funcionado como paliativo para el desarrollo de ansiedad escénica en los casos no clínicos, o si por el contrario ha sido un detonante más, como han concluido estudios como el de White (2012).

Atendiendo a los relatos finales de los participantes se puede concluir que el yoga y la meditación se sintieron como una herramienta para mejorar la regulación emocional y el bienestar personal. Varios afirmaron que las clases de yoga les ayudaron a relajarse, que aplicaron el yoga en algunos momentos estresantes de sus vidas y que las clases de yoga y meditación les sentaban bien.

Estos resultados sobre la práctica de yoga y meditación desde edades tempranas están en consonancia con la información aportada por autores como Thich Nhat Hanh (Hanh \& Plum Village, 2015), Snel (2013), Chanchani y Chanchani (1995) y Kaiser (2013) en sus publicaciones, dirigidas principalmente a padres y profesores con el fin de animarles a practicarlas con regularidad con los niños.

Con relación a las limitaciones que presenta el estudio se puede señalar:

- El reducido número de participantes que no permite generalizar los resultados. 
- La ausencia de grupo de control que no permite comprobar si los resultados fueron debidos al programa o a otros factores externos.

En cuanto a futuras investigaciones que pueden llevarse a cabo continuando esta línea de investigación:

- Se podría desarrollar una investigación durante un periodo de tiempo más prolongado, de varios cursos, con sesiones regulares, para poder evaluar el efecto a largo plazo en el aprendizaje musical.

- Trabajar con una muestra mayor de participantes, incluyendo tal vez al profesorado y a las familias en el proyecto, para que la investigación-acción desarrolle realmente un cambio en la enseñanza.

- Sería idóneo además de las herramientas utilizadas, usar las específicas para medir la ansiedad escénica, validarlas para la población estudiada.

- Incluir un grupo de control para poder realizar comparaciones entre y dentro de los grupos.

- Incluir individuos con clara sintomatología de trastornos musculoesqueléticos.

A pesar de las numerosas limitaciones en este programa piloto preliminar desde la perspectiva de la investigación, los resultados indican que el enfoque utilizado fue bien acogido por el alumnado y que es probable que sea beneficioso tanto para mejorar sus facultades de aprendizaje como para aliviar los problemas que enfrentan como estudiantes de música. El yoga y la meditación pueden suponer un complemento eficaz y rentable en los planes de estudios de los conservatorios, principalmente con relación a la ansiedad escénica. Construir un nuevo modelo de enseñanza y aprendizaje instrumental se convierte no solo en necesario, sino en una realidad con programas como este. Un arte como la música no debería estar nunca asociada al sufrimiento físico o emocional. Crear un aprendizaje sano y feliz entre los estudiantes de música es posible.

\section{REFERENCIAS}

Adams, A. D. (2012). Yoga and Saxophone Performance: The Integration of Two Disciplines. (Doctoral Thesis non publicada). Arizona, EEUU: Arizona State University.

Alajmo, T., Jiménez, L. K., \& Gantiva, C. A. (2013). Evaluación del efecto de un programa basado en mindfulness para el manejo de ansiedad en niños escolarizados. El Astrolabio, 7-19.

Bardin, L. (2008). A análise de conteúdo. Lisboa: Edições 70.

Beaucheim, J., Hutchins, T., \& Patterson, F. (2008). Mindfulness meditation may lessen anxiety, promote social skills, and improve academic performance among adolescents with learning disabilities. Complementary Health Practice Review, 13(1), 34-45.

Bertram, T., \& Pascal, C. (2009). Manual DQP - Desenvolvendo a Qualidade em Parceria. Lisboa: Ministério da Educação. 
Brandfonbrener, A. G., \& Kjelland, J. M. (2002). Music Medicine. In R. Parncutt, \& G. McPherson, The Science \& Psychology of Music Performance: Creative Strategies for Teaching and Learning (pp. 83-96). Oxford: Oxford University Press.

Brown, K. W., \& Ryan, R. M. (2003). The Benefits of Being Present: Mindfulness and Its Role in Psychological Well-Being. Journal of Personality and Social Psychology, 84(4), 822-848.

Carrasco, C. (2016). Mindfulness en Educación (Trabajo Fin de Grado). Badajoz: Universidad de Extremadura.

Cavanagh, K., Strauss, C., Cicconi, F., Griffiths, N., Wyper, A., \& Jones, F. (2013). A randomised controlled trial of a brief online mindfulness-based intervention. Behaviour Research and Therapy, 51, 573-578.

Chanchani, S., \& Chanchani, R. (1995). Yoga for children - A Complete llustrated Guide to Yoga. New Delhi: UBS Publishers' Distributors.

Chang, J. C., Midlarsky, E., \& Lin, P. (2003). Effects of meditation on music performance anxiety. Medical Problems of Performing Artists, 18(3), 126-130.

Collyer, S. (2018). Yoga for singers - A holistic practice tool. (Doctoral Thesis non publicada), Queensland University of Technology.

Cox, W., \& Kenardy, J. (1993). Performance anxiety, social phobia, and setting effects in instrumental music students. Journal of Anxiety Disorders, 7, 49-60.

Elliot, J. (1990). La investigación acción en educación. Madrid: Morata.

Epply, K. R., Abraham, A. I., \& Shear, J. (1989). Differential effects of relaxation techniques on trait anxiety: A meta-analysis. Journal of Clinical Psychology, 45, 957-974.

Farnsworth-Grodd, V. A. (2012). Mindfulness and the self-regulation of music performace anxiety. Auckland, EEUU: University of Auckland.

Galantino, M. L., Galbavy, R., \& Quinn, L. (2008). Therapeutic effects of yoga for children: A systematic review of the literature. Pediatric Physical Therapy, 20(1), 66-80.

Gallego, J., Aguilar-Parra, J., Cangas, A., Langer, Á., \& Mañas, I. (2017). Effect of a Mindfulness Program on Stress, Anxiety and Depression in University Students. Spanish Journal of Psychology, (17), 1-6. doi:10.1017

Goyette, G., \& Léssard-Hebert, M. (1988). La investigación-acción. Funciones, fundamentos e instrumentación. Barcelona: Laertes.

Hanh, T. N. (2007). El milagro del mindfulness. Barcelona: Omiro.

Hanh, T. N., \& Plum Village, C. (2015). Plantando semillas. La práctica del mindfulness con niños. Barcelona: Kairós.

Harne, A. (2017). Availability for learning: effects of mindfulness meditation on student cognitive abilities. (Doctoral Thesis non publicada), Philadelphia, Philadelphia College of Osteopathic Medicine.

Hoge, E. A., Bui, E., Goetter, E., Robinaugh, D. J., Ojserkis, R. A., Fresco, D. M., \& Simon, N. M. (2015). Change in Decenteting Mediates Improvement in Anxiety in Mindfulness-Based Stress Reduction for Generalized Anxiety Disorder. Cognitive therapy and research, 39(2), 228-235.

lyengar, B. (2017). Luz sobre el yoga. Barcelona: Editorial Kairós.

Kabat-Zinn, J. (2003b). Mindfulness-Based Interventions in Context: Past, Present, and Future. Clinical Psychology: Science and Practice, 10(2), 144-156.

Kabat-Zinn, J., Lipworth, L., \& Burney, R. (1985). The Clinical Use of Mindfulness Meditation for the SelfRegulation of Chronic Pain. Journal of Behavioral Medicine, 8(2), 163-190.

Kabat-Zinn, J., Massion, A. O., Kristeller, J., Peterson, L. G., Fletcher, K. E., Pbert, L., . . Santorelli, S. (1992). Effectiveness of a meditation-based stress reduction program in the treatment of anxiety disorders. American Journal of Psychiatry, 149, 936-943.

Kaiser, S. (2013). El niño atento. Mindfulness para ayudar a tu hijo a ser más feliz, amable y compasivo. Bilbao: Desclée de Brouwer. 
Kenny, D. T. (2004). Music performance Anxiety: is it the music, the performance or the anxiety? Music Forum, 1-7.

Kenny, D. T. (2006). Music Performance Anxiety: Origins, phenomenology, assesment and treatment. Context 31, 51-64.

Kenny, D. T., Davis, P., \& Oates, J. (2004). Music performance anxiety and occupational stress amongst opera chorus artists and their realtionship with state and trait anxiety and perfectionism. Journal of Anxiety Disorders, 18, 757-777.

Kenny, D., \& Osborne, M. (2006). Music performance anxiety: New insights from young musicians. Advances in Cognitive Psychology, 2(2-3), 103-112.

Khalsa, S. B. (2004). Yoga as a therapeutic intervention: A bibliometric analysis of published research studies. Indian Journal of Physiology and Pharmacology, 48(3), 269-285.

Khalsa, S. S., Shorter, S. M., Cope, S., Wyshak, G., \& Sklar, E. (2009). Yoga ameliorates performance anxiety and mood disturbance in young professional musicians. Applied Psychophysiology Biofeedback, 34, 279289.

Khalsa, S., \& Cope, S. (2006). Effets of a yoga lifestyle intervention on performance-related characteristics of musicians: A preliminary study. Med Sci Monit, 12(8), CR325-331.

Khalsa, S., Butzer, B., Shorter, S., Reinhardt, K., \& Cope, S. (2013). Yoga Reduces Performance Anxiety in Adolesncent Musicians. Alternative Therapies, 19(2), 34-45.

Lin, P., Chang, J., Zemon, V., \& Midlarsky, E. (2008). Silent ilumination: A study on Chan (Zen) Meditation, anxiety, and musical performance quality. Psychology of Music, 36(2), 139-155.

López-González, L. (2010). El Programa TREVA (Técnicas de Relajación Vivencial Aplicadas al Aula): aplicaciones, eficacia y acciones formativas. In J. Riart, \& A. Martorell, L'estrès laboral docent (pp. 183196). Barcelona: ISEP.

Mañas, I., Franco, C., Cangas, A. J., \& Gallego, J. (2011). Incremento del rendimiento académico, mejora del autoconcepto y reducción de la ansiedad en estudiantes de Bachillerato a través de un programa de entrenamiento en mindfulness (conciencia plena). Encuentros en Psicología(28), 44-62.

Máximo-Esteves, L. (2008). Visão Panorâmica da Investigação-Acção. Porto: Porto Editora.

Miller, J. J., Fletcher, K., \& Kabat-Zinn, J. (1995). Three-year follow-up and clinical implications of a mindfulness meditation-based stress reduction intervention in the treatment of anxiety disorders. General hospital psychiatry, 17(3), 192-200.

Olson, M. (2009). Musician's Yoga - A guide to Practice, Performance and Inspiration. Boston: Breklee Press.

Orozco, L., \& Solé, J. (2000). Tecnopatías del Músico. Madrid: Ariza Comunicaciones.

Rodrigues, J. R. (2014). Ansiedade em Estudantes de Medicina. Covilhã, Portugal: Universidade da Beira Interior - Ciências da Saúde.

Simón, V. (2011). Aprender a practicar mindfulness. Y abrir el corazón a la sabiduría y la compasión. Barcelona: Sello Editorial.

Snel, E. (2013). Tranquilos y atentos como una rana. Barcelona: Kairós.

Stern, J. R., Khalsa, S. S., \& Hofmann, S. G. (2012). A Yoga Intervention for Music Performance Anxiety in Conservatory Students. Medical Problems of Performing Artists, 27(3), 123-128.

Steyn, B. J., Steyn, M. H., Maree, D. J., \& Panebianco-Warrens, C. (2016). Psychological skills and mindfulness training effects on the psychological wellbeing of undergraduate music students: An exploratory study. Journal of Psychology in Africca, 27(2), 167-171.

Stoeber, J., \& Eismann, U. (2007). Perfectionism in young musicians: Relations with motivation, effort, achievement, and distress. Personality and Individual Differences, 43, 2181-2192.

White, L. S. (2012). Reducing stress in school-age girls through mindful yoga. Journal of Pediatric Health Care, 26(1), 45-56. 
Winter, R. (2003). Buddhism and action research: towards an appropriate model of inquiry for the caring professions. Educational Action Research, 11(1), 141-160.

Xu, H. (2010). Application of Zen Techniques to Overcome Performance Anxiety. (Tesis doctoral non publicada), Florida: The Florida State University College of Music.

Zarza-Alzugaray, F. J., Casanova-López, Ó., \& Orejudo-Hernández, S. (2016a). Ansiedad escénica en estudiantes de piano. ulu, 1, 28-35.

Zarza-Alzugaray, F. J., Casanova-López, Ó., \& Orejudo-Hernández, S. (2016b). Ansiedad escénica y constructos psicológicos relacionados. Estudiantes de cinco conservatorios de música españoles. Revista Internacional de Educación Musical, (4), 13-23.

Zarza-Alzugaray, F. J., Casanova-López, Ó., \& Robles-Rubio, J. E. (2016). Relación entre ansiedad escénica, perfeccionismo y calificaciones en estudiantes del Título Superior de Música. ReiDoCrea, 5, 16-21.

Zelazo, P. D., \& Lyons, K. E. (2012). The potential benefits of minfulness training in early childhood: A developmental social cognitive neuroscience perspective. Child Development Perspectives, 6(2), 154160.

Zoogman, S., Goldberg, S. B., Hoyt, W. T., \& Miller, L. (2015). Mindfulness interventios with youth: A metaanalysis. Mindfulness, 6(2), 290-302.

Zung, W. (1971). A Rating Instrument for Anxiety Disorders. Psychossomatics, 6, 371-379. 